\title{
1 mm Dual-polarization Science with CARMA
}

\author{
Charles L. H. Hull ${ }^{1}$, Richard Plambeck ${ }^{2}$, and Gregory Engargiola ${ }^{3}$ \\ ${ }^{1}$ Radio Astronomy Laboratory, University of California, Berkeley. 601 Campbell Hall, Berkeley, CA 94720, USA. \\ TEL: +1-510-642-5275. FAX: +1-510-642-3411. EMAIL: chat@astro.berkeley.edu. \\ 2 plambeck@astro.berkeley.edu \\ ${ }^{3}$ greg@astro.berkeley.edu
}

\begin{abstract}
We have constructed dual polarization $1 \mathrm{~mm}$ receivers for the CARMA array. A key science goal for these receivers is to map the linearly polarized thermal emission from dust grains in interstellar clouds, which may be used to infer the magnetic-field morphologies in these regions. Polarization fractions of a few percent are typical of this radiation. For an aperture synthesis array like CARMA it is advantageous to measure this weak linear polarization by cross-correlating the signals from circularly polarized feeds. To observe circular polarization, we install a waveguide polarizer between the feed horn and the OMT (orthomode transducer), at a temperature of $4 \mathrm{~K}$, to convert incoming circularly polarized signals into linearly polarized signals. The receivers use waveguide OMTs to split the incoming radiation into orthogonal linear polarizations. The two OMT outputs are coupled to separate SIS (superconductorinsulator-superconductor) mixers and WBA-13 LNAs (low-noise amplifiers).
\end{abstract}

\section{Introduction}

CARMA (the Combined Array for Research in Millimeter-wave Astronomy) is an aperture-synthesis telescope in Cedar Flat, CA that is operated by Caltech, UC Berkeley, the University of Illinois at Urbana-Champaign, the University of Maryland, and the University of Chicago (1). It is a 23-element heterogeneous array, comprising six 10-meter, nine 6-meter, and eight 3.5-meter antennas. The 6- and 10-meter dishes currently observe in the 1 and $3 \mathrm{~mm}$ bands; soon all 23 antennas will operate at both $3 \mathrm{~mm}$ and $1 \mathrm{~cm}$.

With the installation of the $1 \mathrm{~mm}$ dual-polarization system described herein, CARMA will be able to access the wealth of polarization information contained in observed $1 \mathrm{~mm}$ radiation. Of particular interest is the linearly polarized emission from dust grains in interstellar clouds, which are bright at $1 \mathrm{~mm}$. Mapping polarization will allow us to probe the morphology of magnetic fields in those regions-in particular, in the collapsing envelopes and extended outflows around deeply embedded Class 0 protostellar cores-and to identify scientifically interesting targets that can be followed up with the unprecedented resolution and sensitivity of ALMA.

Figure 1 shows a photo of one of the $1 \mathrm{~mm}$ dual-polarization receivers that is mounted in the dewars on the 10 - and 6-m telescopes at CARMA. The receiver consists of a feed horn; a waveguide circular-to-linear polarizer; a waveguide orthomode transducer (OMT); two mixers with superconductor-insulator-superconductor (SIS) devices designed by the NRAO for ALMA's band 6 (2); and two wide-band, low-noise WBA13 intermediate frequency (IF) amplifiers. The entire module is installed in a dewar, the innermost stage of which is cooled to $4 \mathrm{~K}$.

In the following sections, we give brief descriptions of the various components of the $1 \mathrm{~mm}$ dual-polarization receivers.

\section{The polarizer}

The waveguide polarizer we use converts circularly polarized light to linearly polarized light. It has a 2-section design, using half-wave and quarter-wave retarder sections rotated axially by $59.5^{\circ}$ with respect to one other to achieve broadband performance (3). The design was inspired by the broadband waveguide polarizer described by Kovac and Carlstrom (4) and by Lilie (5), and is discussed in detail by Plambeck (6). Figure 2 shows the mandrel that was used 


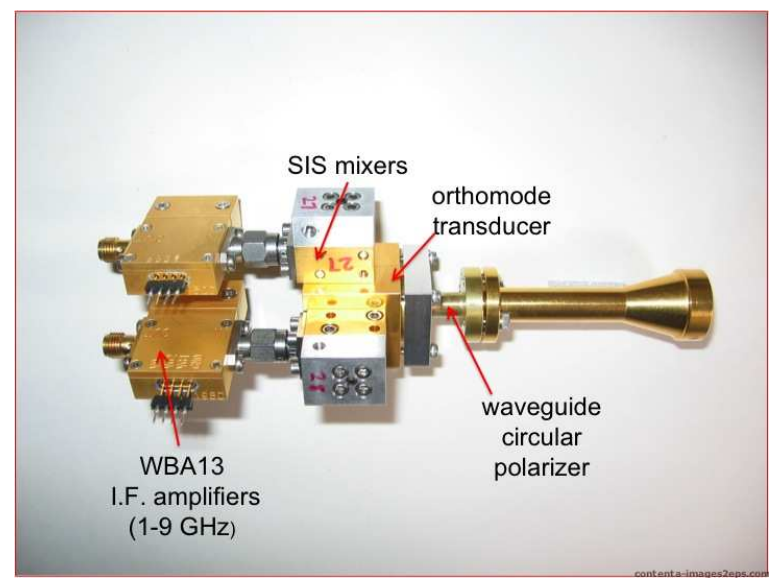

Figure 1: Left: a $1 \mathrm{~mm}$ dual-polarization receiver.

as a mold for the inside of the polarizer, as well as an HFSS simulation showing the transition from circular to linear polarization as radiation passes through the polarizer from right to left.
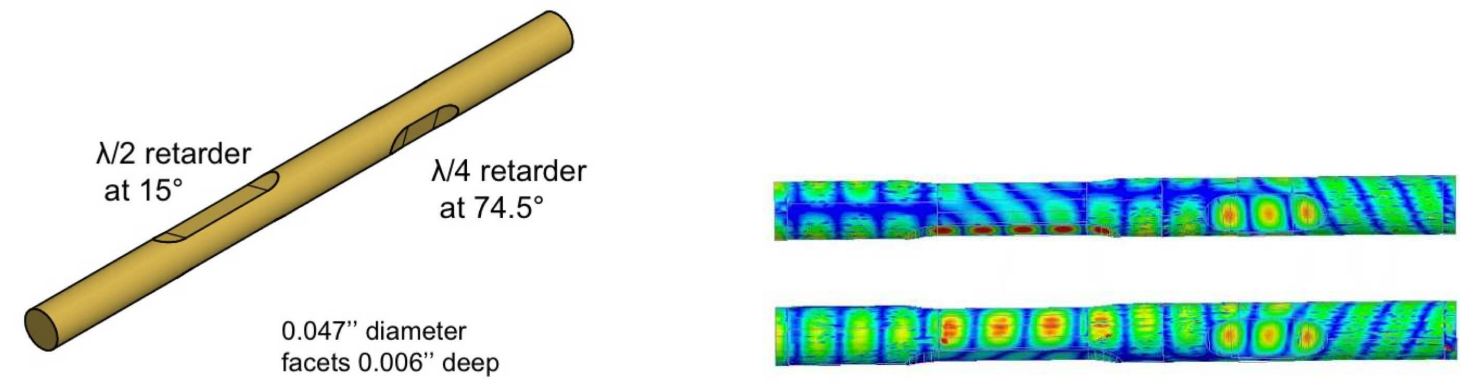

Figure 2: Left: the mandrel, which was used as a mold for the inside of the polarizer; the two flats are separated by an angle of $59.5^{\circ}$, and act as a wideband circular-to-linear converter. Right: the HFSS simulation showing radiation being converted from circular to linear polarization as it propagates from right to left.

Any polarizer will experience some leakage of radiation between the two polarizations. For example, the leakage between left-circular and right-circular can be expressed as $V_{r}^{\prime}=V_{r}+D_{r} V_{l}$, where $V_{r}^{\prime}$ is the voltage measured by the right-circular receiver; $V_{r}$ and $V_{l}$ are the ideal voltages of the right- and left-circular receivers, respectively; and $D_{r}$ is the leakage term dictating how much signal leaks from left-circular to right-circular. The theoretical leakages for polarizers with one, two, and three sets of flats are plotted on the left in Figure 3. While the leakages for the threesection polarizer are obviously preferable, the story is different when the simulations include reasonable machining tolerances of $0.0003 "$ in the depth of the flats and $0.2^{\circ}$ in the angle between the flats. The results of the simulation clearly favor the two-section polarizer, which is the design we use in our receivers.

\section{The orthomode transducer (OMT)}

After being converted in the polarizer from circular to linear polarization, the radiation enters the OMT, which splits the radiation into its two orthogonal linear components, each of which are fed into different sets of mixers and amplifiers. Figure 4 shows a turnstile-junction OMT, which we fabricated using four blocks (7).

Many of our OMTs behave well across the $1 \mathrm{~mm}$ band; however, many exhibit resonances like those in the top plot 

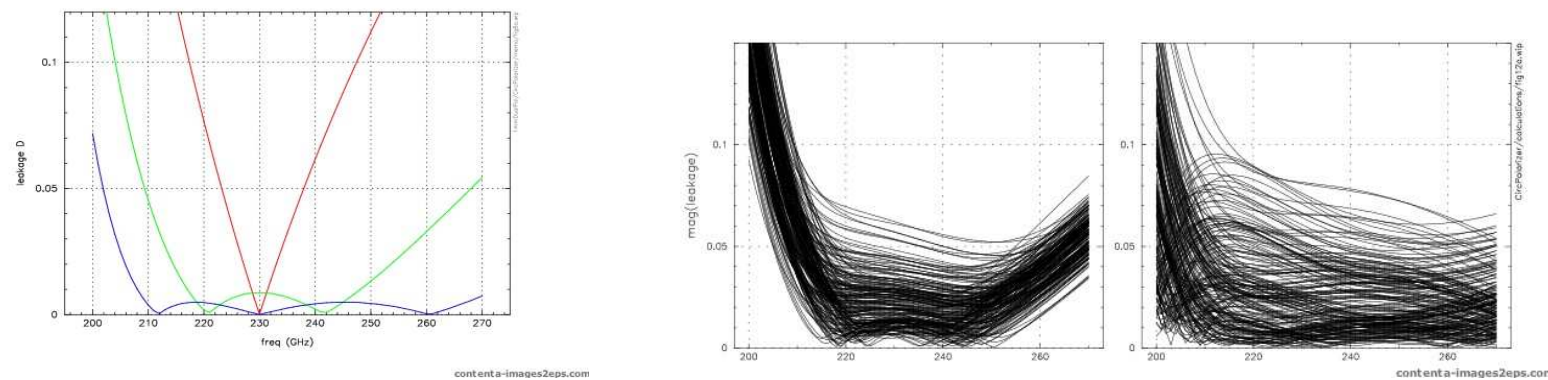

Figure 3: Left: the theoretical leakage percentage as a function of frequency using polarizers with one (red), two (green), and three (blue) sets of flats. Center: leakages for the two-section polarizer, simulated using machining errors of $0.0003 "$ in the depth of the flats and $0.2^{\circ}$ errors in the angle between the flats. Right: leakages for the three-section polarizer using the aforementioned machining errors.

on the right-hand side of Figure 4. Simulations show that these resonances are probably due to fabrication errors that cause misalignments in one or more of the four quarters that make up the OMT block. These misalignments change the electrical path length of the waveguide between the turnstile junction and one of the power combiner inputs. This in turn causes a phase difference between the sidearms of the turnstile junction, which gives rise to reflections in the power combiner (8).
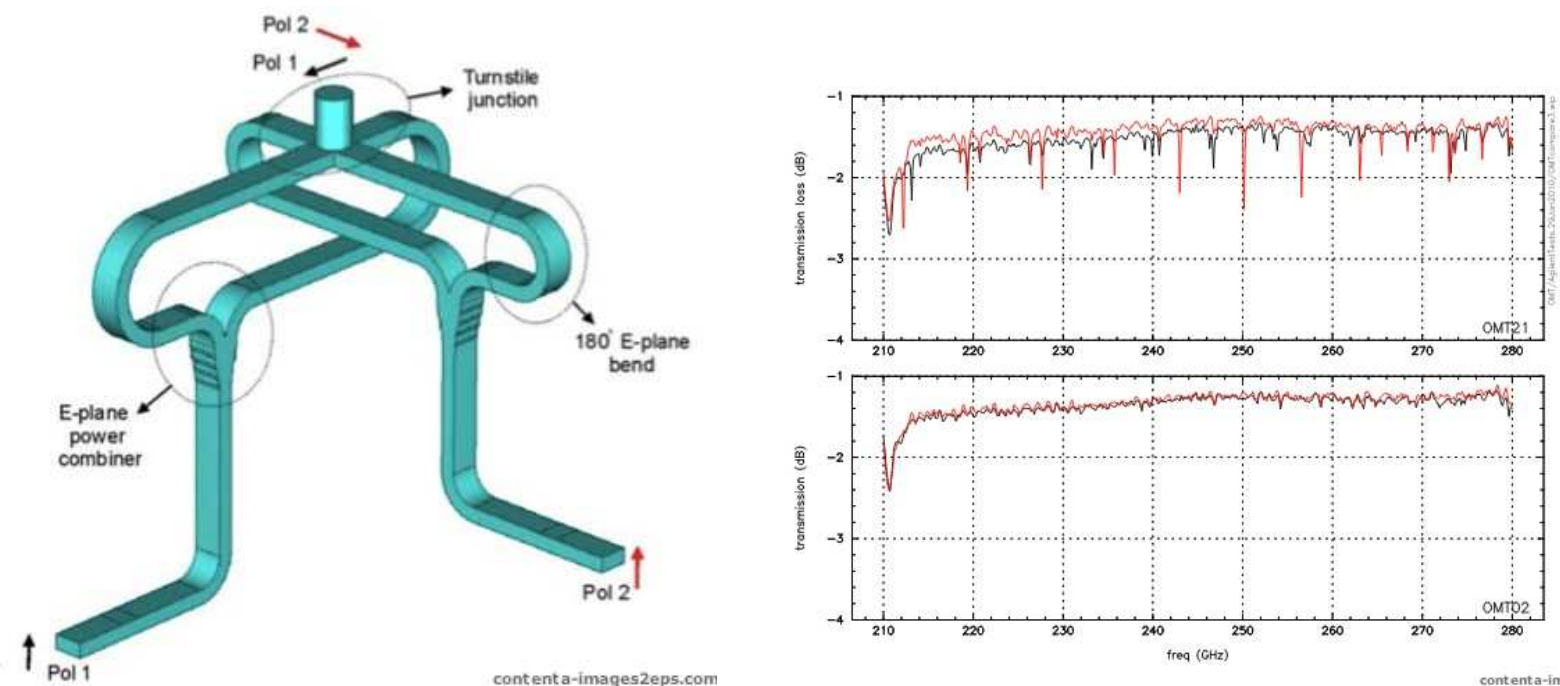

Figure 4: Left: a diagram of the waveguides inside the OMT. Right: passbands for a poorly behaved OMT with resonances (top) and a well behaved OMT without them (bottom).

\section{Conclusion}

The novel $1 \mathrm{~mm}$ receivers described above will allow CARMA to probe the polarization of regions in cold, dense molecular clouds that harbor nascent stars. Peering into these enshrouded regions will help us discern how important a role magnetic fields play in the earliest epoch of star formation, which has hitherto been poorly understood. We expect to use CARMA to map the polarization in a large sample of protostellar cores, and hope to identify particularly interesting sources for even higher-sensitivity follow-up with ALMA. 


\section{Acknowledgements}

Support for CARMA construction was derived from the Gordon and Betty Moore Foundation, the Kenneth T. and Eileen L. Norris Foundation, the James S. McDonnell Foundation, the Associates of the California Institute of Technology, the University of Chicago, the states of California, Illinois, and Maryland, and the National Science Foundation.

Ongoing CARMA development and operations are supported by the National Science Foundation under a cooperative agreement, and by the CARMA partner universities. C.L.H.H. is supported by an NSF Graduate Fellowship.

\section{References}

[1] D. Bock, A. D. Bolatto, D. W. Hawkins, A. J. Kemball, J. W. Lamb, R. L. Plambeck, M. W. Pound, S. L. Scott, D. P. Woody, and M. C. H. Wright, "First results from CARMA: the combined array for research in millimeterwave astronomy," in Society of Photo-Optical Instrumentation Engineers (SPIE) Conference Series, vol. 6267, July 2006.

[2] A. R. Kerr, S.-K. Pan, A. W. Lichtenberger, N. Horner, J. E. Effland, and K. Crady, "A single-chip balanced SIS mixer for 200-300 GHz," ALMA Memo Series, no. 308, pp. 1-9, 2000. [Online]. Available: http://www.alma.nrao.edu/memos/html-memos/alma308/memo308.pdf

[3] S. Pancharatnam, "Achromatic combinations of birefringent plates: Part ii. an achromatic quarter-wave plate," Memoirs of the Raman Research Institute, Bangalore, no. 71, pp. 137-144, March 1955.

[4] J. M. Kovac and J. E. Carlstrom, "Broadband high precision circular polarizers and retarders in waveguides," US Patent 6963253 B2, November 2005.

[5] P. Lilie, "Wide-Band Circular Polarizers Made with Quarter-Wave and Half-Wave Plates," ATNF Technical Memos, no. 39.3/106, pp. 1-7, 2001. [Online]. Available: http://www.atnf.csiro.au/observers/memos/AT39.3_106.pdf

[6] R. Plambeck and G. Engargiola, "Circular Polarizers for the CARMA 1mm Receivers," CARMA Memos, no. 54, pp. 1-35, 2010. [Online]. Available: http://www.mmarray.org/memos/carma_memo54.pdf

[7] A. Navarrini and R. Plambeck, "A turnstile junction waveguide orthomode transducer," IEEE Transactions on Microwave Theory and Techniques, vol. 54, no. 1, pp. 272 -277, 2006.

[8] A. Navarrini, A. Bolatto, and R. L. Plambeck, "Preliminary test results of the turnstile junction waveguide orthomode transducer for the $1 \mathrm{~mm}$ band," CARMA Memos, no. 32, pp. 1-19, 2006. [Online]. Available: http://www.mmarray.org/memos/carma_memo32.pdf 